\title{
Current state of transcatheter mitral valve implantation in bioprosthetic mitral valve and in mitral ring as a treatment approach for failed mitral prosthesis
}

\author{
Vratika Agarwal $^{1 \#}$, Ryan K. Kaple ${ }^{1 \#}$, Hetal H. Mehta ${ }^{1}$, Prabhjot Singh ${ }^{1}$, Vinayak N. Bapat ${ }^{2}$ \\ ${ }^{1}$ Section of Cardiovascular Medicine, Department of Medicine, Yale School of Medicine, New Haven, Connecticut, USA; ${ }^{2}$ Division of Cardiothoracic \\ Surgery, The Minneapolis Heart Institute Foundation, Minneapolis, Minnesota, USA \\ \#These authors contributed equally to this work. \\ Correspondence to: Vratika Agarwal, MD. Department of Medicine, Section of Cardiovascular Medicine, Yale School of Medicine, Courier 333 Cedar \\ Street, Office 345 Dana, New Haven, CT 06520, USA. Email: Vratika.agarwal@yale.edu; Ryan K. Kaple, MD. Department of Medicine, Section of \\ Cardiovascular Medicine, Yale School of Medicine, Courier 333 Cedar Street, Office 321 Dana, New Haven, CT 06520, USA. \\ Email: Ryan.kaple@yale.edu.
}

\begin{abstract}
With heightened awareness of mitral valve disease and improvement in surgical techniques, the use of mitral valve bioprostheses has increased. There is a large aging population with prior surgical valvular interventions. Limited durability of the prosthesis due to valvular degeneration over time may necessitate the need for repair or replacement of the prior prosthesis in the future. This usually entails another surgical intervention in this population with elevated risk for a reoperation. There is an ongoing clinical need for newer, less invasive options that are feasible and carry a lower complication rate. The advent of transcatheter heart valve (THV) therapies has opened up a wide range of therapeutic options for treatment of a failed bioprosthesis. Their safety and feasibility are now well established. This article serves as a review of the currently available THVs for implantation in the mitral position, the pre-procedural assessment, the challenges associated with implantation, as well as outcomes associated with a mitral valve-in-valve (VIV) and a mitral valve-in-ring (VIR) procedure.
\end{abstract}

Keywords: Valve-in-valve (VIV); valve-in-ring (VIR); mitral valve bioprosthesis; surgical heart valve; transcatheter mitral valve replacement (TMVR); transcatheter heart valve (THV)

Submitted May 21, 2021. Accepted for publication Aug 25, 2021.

doi: 10.21037/acs-2021-tviv-80

View this article at: https://dx.doi.org/10.21037/acs-2021-tviv-80

\section{Introduction}

The incidence of valvular heart disease has increased substantially in the past decade. In developing countries, rheumatic heart disease still remains the principal cause of valvular pathology. In industrialized countries, the primary etiology of valvular heart disease is valve degeneration, mainly driven by an aging population (1-3). Mitral regurgitation (MR) is the most common valvular heart disease diagnosed in the United States. In the year 2000, an estimated 2-2.5 million people were diagnosed with significant MR (moderate-to-severe or severe). This number is expected to double by the year 2030 (3). This however may not be a true reflection of the disease burden as a large number of patients remain underdiagnosed. More recently, epidemiologic studies conducted in Olmstead County, Rochester, MN, USA suggest that mitral valve regurgitation is prevalent in approximately $1.0 \%$ of the population, with moderate-to-severe MR accounting for half of those cases $(3,4)$. Given these data, it is apparent that mitral valve disease poses an important public health problem.

The standard of care for most patients with valvular heart disease is either a surgical repair or a surgical replacement if operative risk is favorable. Despite increases in the frequency of mitral valve interventions (5), patients 
with mitral valve disease continue to be undertreated. Patients are often not offered surgery due to lack of therapy awareness on the part of the referring cardiologist or because of excessive surgical risk in patients who have had a delayed referral to a surgeon. The frequency of mitral valve surgery in an Olmstead County cohort for patients who met indications for surgery was a mere $15 \%$. The emergence of improved and more standardized imaging, along with the development of novel transcatheter therapies, provides some hope to correct this historical shortcoming.

\section{Mitral valve anatomy}

Mitral valve apparatus comprises of the mitral valve annulus, anterior and posterior mitral leaflets, medial and lateral commissure, chordae tendineae and the papillary muscles. These structures work synchronously during systole and diastole to accommodate the stress of the blood flow into the ventricle during diastole and the high closing pressure during systole. Changes in the valvular morphology can disrupt the mechanical integrity and lead to valvular dysfunction that manifests as regurgitation or stenosis.

Surgical therapies include leaflet repair, often accompanied with annuloplasty devices that target the annulus, or complete replacement using surgical prosthetic valves. There are two main types of prosthetic heart valves bioprosthetic and mechanical valves. The primary benefit of bioprosthetic valves is the ability to avoid anticoagulation. However, their durability remains a potential limitation, particularly in younger patients. The past few decades have seen a steady rise in the usage of bioprosthetic valves compared to mechanical valves, with rates of bioprostheses increasing from $16.7 \%$ in 1996 to over 50\% in $2013(5,6)$. Nevertheless, the increased use of bioprosthetic mitral valves has not necessarily resulted in improved outcomes. In patients under seventy, prosthetic valves have been associated with higher mortality and reoperation rates. Mechanical valves, on the other hand, are associated with higher rates of stroke, and bleeding due to the need for lifelong anticoagulation (7).

As the life span of the average American increases, there has also been an escalation in the incidence of prosthetic valve deterioration in the elderly population. With improvement in operative techniques, the mortality and morbidity associated with reoperation in this elderly population is lower than what was seen in the past, but is still noted to range from $7-15 \%(8-10)$. Advanced age ( $>65$ years), renal insufficiency, advanced New York Heart
Association (NYHA) class, and non-elective operations are the main factors that are associated with increased hospital mortality in reoperative surgery. This increased risk associated with reoperation makes transcatheter therapies an appealing option in this population.

\section{Devices for surgical mitral valve repair}

Surgical techniques used to repair or replace the mitral valve continue to evolve. Surgical repair can now be performed via right thoracotomy or minimally invasive robotic techniques rather than a full sternotomy in selected patients. Mitral valve repair, rather than replacement, is the preferred therapy for isolated primary MR. Mitral Regurgitation International Database (MIDA) registry, encompassing thirty-five years of data, suggests repairs account for a large majority (89\%) of mitral valve surgeries (11). The choice of repair versus mitral valve replacement (MVR) seems to be independent of patient age, as the median age for mitral repair patients was similar to that for MVR patients (65 versus 67, respectively). The strong preference for mitral repair over MVR is supported by the registry data, with repair patients doing far better than their MVR counterparts with degenerative MR. In the MIDA registry, mitral valve repair resulted in both a lower operative mortality and a higher 20-year survival rate. The operative mortality following mitral valve repair was $1.3 \%$ compared to $4.7 \%$ for MVR. At 20 years, the survival rate for mitral repair patients was nearly twice the survival rate after MVR (46\% versus 23\%, respectively). This mortality benefit, however, is less apparent in ischemic MR (12). Similarly, the complication rate for mitral repair is lower when compared to MVR, with complications predominantly including stroke, endocarditis and bleeding. However, both mitral valve repair and replacement are similar in terms of recurrent MR, with rates being less than $5 \%$.

Mitral valve repair for degenerative MR often involves resection of the degenerated or redundant leaflet and primary repair of this area. Chordal shortening, chordal transfer and commissural annuloplasty are being performed less frequently. Insertion of Gore-Tex neochordae to support and suspend the prolapsing segment has now become a standard surgical approach. The repair is often supplemented with annuloplasty using either an annuloplasty band or ring. In fact, all mitral valve repairs in the prospective MIDA database for degenerative MR were supplemented with annuloplasty rings. There were geographic differences in the type of devices used, with 
European centers preferring semi-rigid and rigid rings, and US centers favoring flexible annuloplasty bands (11).

Bands and rings can be either complete, supporting the entire circumference of the annulus, or incomplete, supporting the annulus only partially. The primary difference between the surgical band and ring is flexibility or ability to conform to the given shape of the annulus. Bands are made of flexible material and surgical rings can either be rigid or semi-rigid. Choice of band versus ring depends on surgeon and institutional preferences. Type of annuloplasty device plays a crucial role in deciding candidacy for future valve-in-ring (VIR). As the options for transcatheter therapies continue to broaden, it is important for the implanting surgeon to think about not only getting the best result for the patient at the current time, but to also consider possible future options for the patient if the prosthesis fails. Individual characteristics of currently approved surgical annuloplasty devices are noted in Table 1.

Several transcatheter therapies for MR have emerged as alternatives to surgery. Transcatheter edge-to-edge repair (TEER) is effective in reducing symptoms and allowing for left ventricular remodeling with two to three grade reduction in $\mathrm{MR}$, and was demonstrated to be equivalent to surgery in patients who are at high or prohibitive risk in terms of improvement of MR and NYHA class symptoms (13-15). Consequently, TEER has earned a class IIa indication for treating patients with primary MR who are considered to be at elevated risk for surgery per the recent American College of Cardiology and American Heart Association (ACC/AHA) valve guidelines (16). More recently, TEER was shown to improve symptoms, prolong life and reduce hospitalization in appropriately selected patients with severe secondary MR and reduced ejection fraction (17). Transcatheter mitral valve replacement (TMVR) is being evaluated in the global multicenter APOLLO (Intrepid Valve) and SUMMIT (Tendyne Valve) trials, currently testing the safety and efficacy of this therapy.

\section{Surgical bioprosthetic valves}

Bioprosthetic valves can be classified as xenografts, homografts or autografts. Xenografts are animal derived, chemically treated tissue valves that can be bovine/porcine or equine. The tissue used is from the animal's cardiac valve, pericardium or jugular venous valve. Transcatheter valves are also grouped into xenografts. Homografts are derived from humans, either a cadaver or a live donor during heart transplantation. Finally, an autograft involves transplantation of the patient's own valve from one position to another.

There are three main components of a surgical bioprosthetic valve: valve leaflets, the stent frame and the suture ring. The valve leaflets are often sewn in the inner aspect of the stent frame. The exception to this is the Mitroflow (Sorin Group USA Inc, Arvada, CO, USA), Trifecta (St Jude Medical Inc, Saint Paul, MN, USA) and the Dokimos (Labcor, Belo Horizonte, Brazil) valves. In these valves, the tissue leaflets are sutured on the outside of the stent to allow for better valve area and hemodynamics. The characteristics of the surgical heart valves used in the mitral position are described in Table 2.

Stentless valves lack a stent frame and are composed of only valvular tissue and the sewing ring. They are not used routinely in the mitral position. Sutureless valves are valves that have a stent frame that anchors in the aortic root (rather than a sewing ring) and thus are not used in the mitral position.

Choosing the optimal valve for each patient requires a thorough work up and a detailed discussion with the patient regarding the risks and benefits associated with each valve type. Mechanical valves are noted to be more durable but require lifelong anticoagulation. Tissue valves on the other hand do not require lifelong anticoagulation but are subject to structural valve deterioration (SVD).

\section{Understanding the dimensions of the prosthesis}

Unlike the aortic valve, mitral valve is not a circular structure. Rather, the mitral annulus is a complex asymmetric fibrous structure that has a $3 \mathrm{D}$ saddle shape and exhibits geometrical changes throughout the cardiac cycle. The saddle shaped annulus is described as having an anterior and posterior horn which are the two most atrial segments along the annulus. The anterior horn continues as a fibrous ridge towards the aortic valve and constitutes the intervalvular fibrosa, commonly referred to as the "aortomitral continuity". The anterior horn remains stable in shape during systole and diastole. The base of the two horns is formed by medial and lateral trigones. These are fibrous triangular structures at the inflection of the mitral annulus. The distance between the medial and lateral trigones is known as the intertrigonal distance (IT). The valve comprises of a longer anterior leaflet and a shorter posterior leaflet which is noted to have scalloped indentations. The distance between the two ends/ commissures is known as the commissure to commissure distance (CC). The distance between the anterior and 
Table 1 Properties of commercially available mitral valve annuloplasty bands and rings

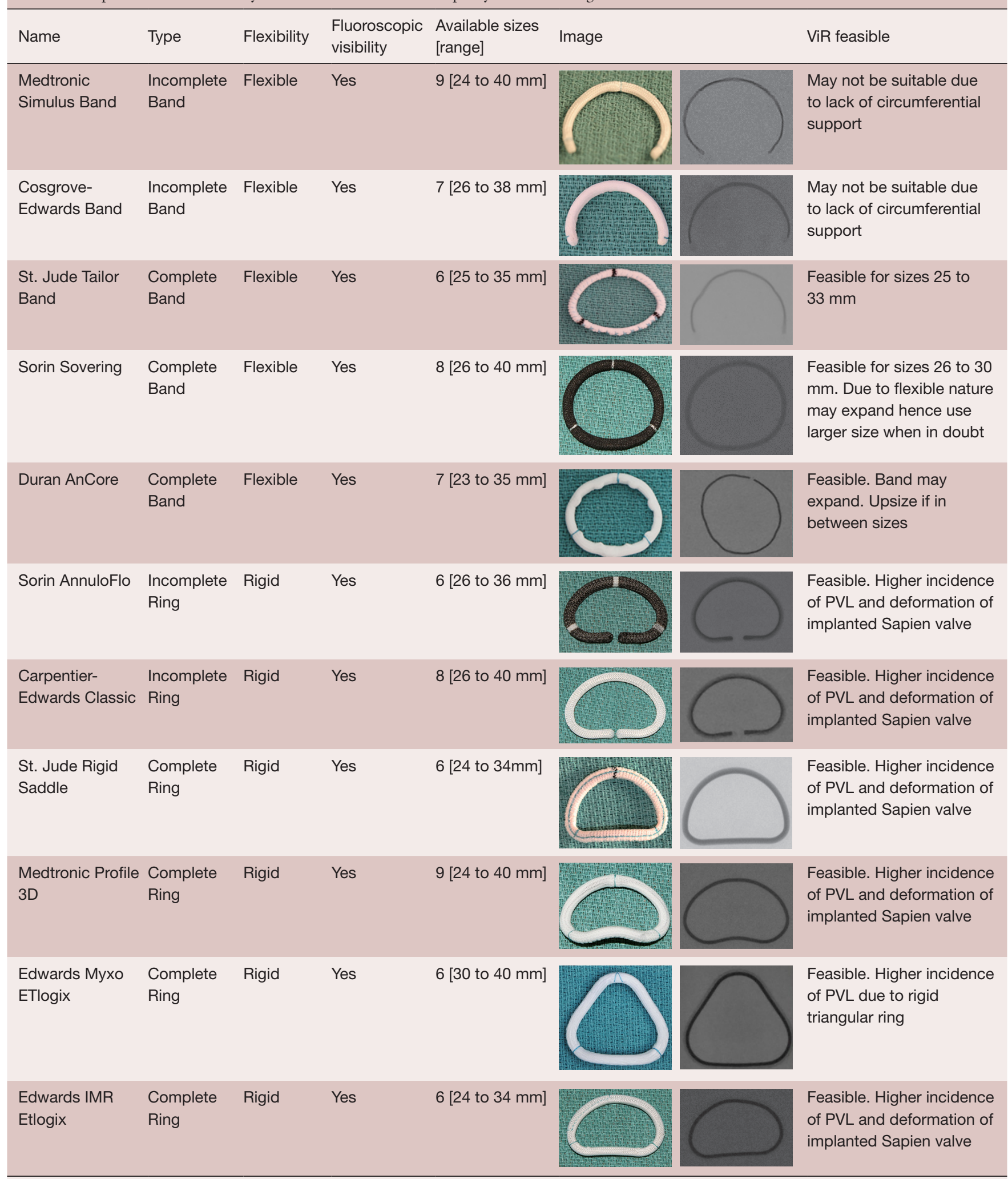

Table 1 (continued) 


\begin{tabular}{|c|c|c|c|c|c|c|}
\hline Name & Type & Flexibility & $\begin{array}{l}\text { Fluoroscopic } \\
\text { visibility }\end{array}$ & $\begin{array}{l}\text { Available sizes } \\
\text { [range] }\end{array}$ & Image & ViR feasible \\
\hline $\begin{array}{l}\text { Edwards } \\
\text { GeoForm }\end{array}$ & $\begin{array}{l}\text { Complete } \\
\text { Ring }\end{array}$ & Rigid & Yes & 4 [26 to $32 \mathrm{~mm}]$ & & $\begin{array}{l}\text { Higher incidence of PVL } \\
\text { due to the saddle shaped } \\
\text { ring }\end{array}$ \\
\hline St. Jude Séguin & $\begin{array}{l}\text { Complete } \\
\text { Ring }\end{array}$ & Semi-rigid & No & 9 [24 to $40 \mathrm{~mm}]$ & & $\begin{array}{l}\text { Feasible. Needs } \\
\text { echocardiographic } \\
\text { guidance due to } \\
\text { inadequate visualization } \\
\text { with fluoroscopy }\end{array}$ \\
\hline Sorin AnnuloFlex & $\begin{array}{l}\text { Complete } \\
\text { Ring }\end{array}$ & Semi-rigid & Yes & 6 [26 to $36 \mathrm{~mm}]$ & & Feasible \\
\hline Sorin Memo 3D & $\begin{array}{l}\text { Complete } \\
\text { Ring }\end{array}$ & Semi-rigid & Yes & 8 [24 to $38 \mathrm{~mm}]$ & & Feasible \\
\hline $\begin{array}{l}\text { Medtronic } \\
\text { Simulus }\end{array}$ & $\begin{array}{l}\text { Complete } \\
\text { Ring }\end{array}$ & Semi-rigid & Yes & 9 [24 to $40 \mathrm{~mm}$ ] & & Feasible \\
\hline $\begin{array}{l}\text { Medtronic CG } \\
\text { Future }\end{array}$ & $\begin{array}{l}\text { Complete } \\
\text { Ring }\end{array}$ & Semi-rigid & Yes & 8 [24 to $38 \mathrm{~mm}]$ & & Feasible \\
\hline $\begin{array}{l}\text { Edwards } \\
\text { Physio II }\end{array}$ & $\begin{array}{l}\text { Complete } \\
\text { Ring }\end{array}$ & Semi-rigid & Yes & 9 [24 to $40 \mathrm{~mm}$ ] & & Feasible \\
\hline $\begin{array}{l}\text { Edwards } \\
\text { Physio I }\end{array}$ & $\begin{array}{l}\text { Complete } \\
\text { Ring }\end{array}$ & Semi-rigid & Yes & 9 [24 to $40 \mathrm{~mm}]$ & & Feasible \\
\hline
\end{tabular}

Adapted from valve-in-valve application. VIR, valve-in-ring; PVL, paravalvular leak.

posterior edge is known as the septolateral distance (SL) (Figure 1).

\section{Annuloplasty ring/band dimensions}

Rings and bands are sized by the surgeon using manufacturer provided ring/band sizers that are inserted into the annulus at the time of the operation. The strategy for sizing is dependent largely on the underlying pathophysiology of MR (functional versus primary degenerative pathology). The sizing is based on the CC distance for most even numbered annuloplasty device sizes, and for odd numbered devices the sizing is mostly based on IT distance (18). Table 3 demonstrates the conventional placement of complete and incomplete annuloplasty bands and rings during mitral valve repair. 
Table 2 Properties of commercially available surgical bioprosthetic heart valves used in the mitral position and depiction of alignment of transcatheter heart valve within a failed mitral bioprosthesis

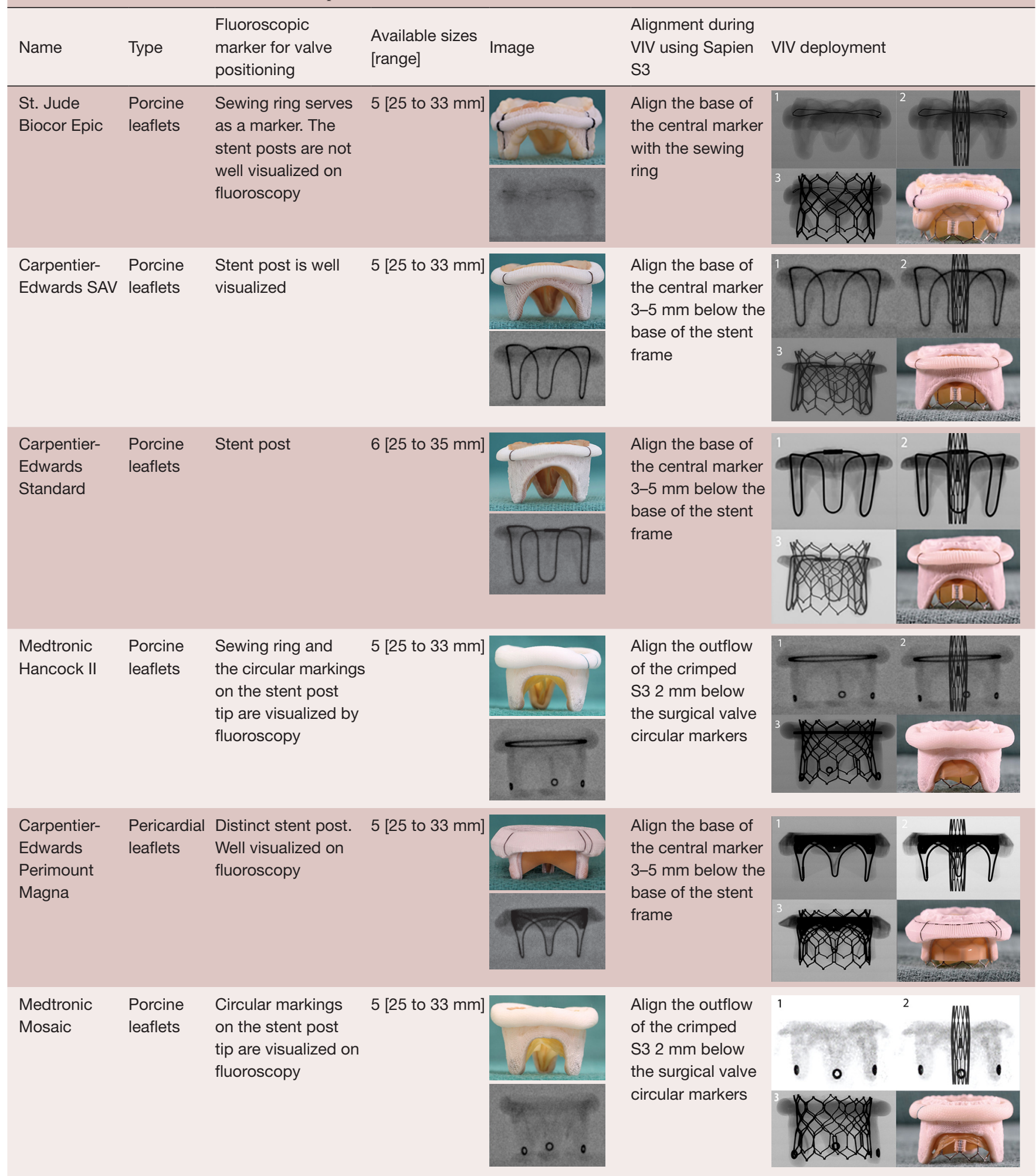

Table 2 (continued) 
Table 2 (continued)

\begin{tabular}{lllll} 
Name & Type & $\begin{array}{l}\text { Fluoroscopic } \\
\text { marker for valve } \\
\text { positioning }\end{array}$ & $\begin{array}{l}\text { Available sizes } \\
\text { [range] }\end{array}$ & $\begin{array}{l}\text { Alignment during } \\
\text { VIV using Sapien } \\
\text { S3 }\end{array}$ \\
\hline $\begin{array}{l}\text { Sorin } \\
\begin{array}{l}\text { Pericarbon } \\
\text { More }\end{array}\end{array}$ & $\begin{array}{l}\text { Pericardial } \\
\text { leaflets }\end{array}$ & $\begin{array}{l}\text { Sewing ring is } \\
\text { visualized on } \\
\text { fluoroscopy }\end{array}$ & $8[19$ to $33 \mathrm{~mm}]$ & $\begin{array}{l}\text { Align the base of } \\
\text { the central marker } \\
\text { with the sewing } \\
\text { ring }\end{array}$
\end{tabular}

Images adapted from VIV application. For optimal valve function the target depth of implantation should not be more than $20 \%$ at the atrial aspect. VIV, valve-in-valve.
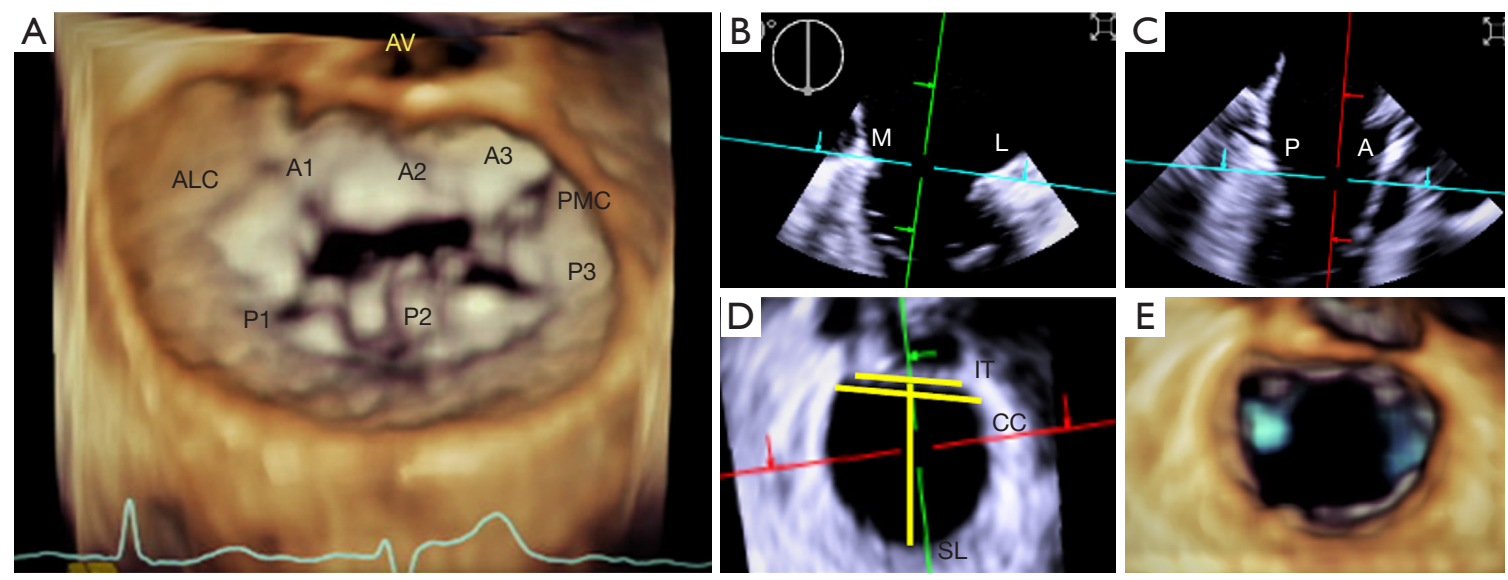

Figure 1 (A)Three-dimensional imaging of the mitral valve displaying the mitral valve en face in surgeon's view. (B-E) Multiplanar reconstruction of the mitral valve at the level of the mitral valve annulus in mid diastole where the commissure to commissure measurement as well as the septolateral or the anterior-posterior measurement is done. AV, aortic valve; CC, commissure to commissure distance; SL, septolateral distance; IT, intertrigonal distance; ALC, anterolateral commissure; PMC, posteromedial commissure.

\section{Bioprosthetic valve dimensions}

Choosing the right size and type of valve for the right patient is an essential step in optimization of outcomes associated with mitral valve replacement. The goal is to select a prosthesis model that provides the largest possible effective orifice area (EOA) with the best hemodynamic performance. This helps in preventing patient prosthesis mismatch (PPM) as well as early SVD. The hemodynamic performance is superior in valves that provide the best valve area. The newer generation valves are notably better when compared to the older generation. Valves with leaflets mounted on the outside of the stent frame provide improved hemodynamic performance when compared to those with leaflets mounted in the interior of the stent frame. Thus, stentless valves are superior to stented valves. Surgical valves are also sized at the time of surgery using sizers. The subvalvular chordae are preserved as much as possible to maintain the left ventricular geometry after the surgery.

It is crucial to note that the manufacturer provided size, based on the sizer, is not the true dimension of the valve. There is no standardization of surgical heart valve sizing across 


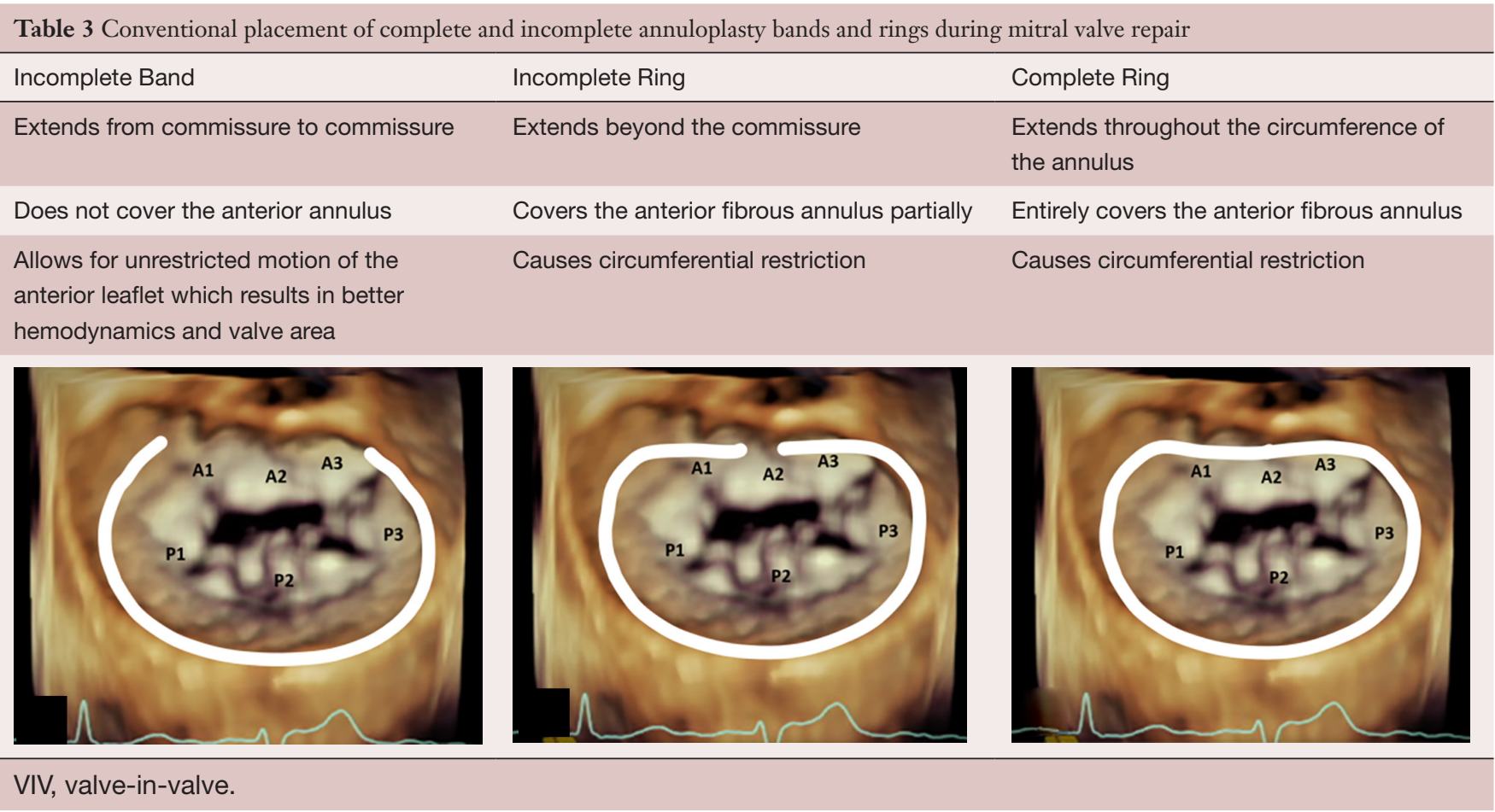

manufacturers (19), and valve size labeling may correspond to either the internal or external valve diameters (20). Two different surgical bioprostheses may have distinct internal and external sewing ring diameters despite having the same label size. For VIV therapy, the most relevant parameter relates to valve internal dimensions, which are often significantly smaller than the labeled valve size. Therefore, when planning a VIV procedure, it is imperative that the heart team determine the precise diameters of the failing bioprosthetic valve. Valve specifications are available from the manufacturer, through published tables generated by independent researchers $(20,21)$, or by consulting the VIV online applications (22). The two key dimensions to identify are the stent internal dimension (Stent ID) and the true internal dimension (True ID). Stent ID is the internal dimension of a bare stent covered with fabric or pericardium without accounting for the effect of artificial leaflets sutured within the stent (23). This is the dimension that is listed by the manufacturer as the model size. True ID is the internal dimension of the valve after the leaflets are mounted within the stent frame (Figure $2 A$ ).

\section{Bovine pericardial versus porcine valves}

The incidence in use of pericardial valves has increased in the past few decades. This is mainly attributed to studies showing that bovine valves tend to provide better hemodynamics and valve area post-procedure. Porcine valves, in contrast, have higher post-operative gradients (particularly true for smaller valves $\leq 21 \mathrm{~mm}$ ). However, more contemporary stent assembly and leaflet mounting in porcine valves have helped in improving valve performance. The True ID for porcine valve with leaflets mounted within the stent frame is smaller when compared to pericardial valves (Figure 2B,2C).

Pathological processes like calcification or pannus can further generate a discrepancy between the expected and the observed internal stent diameters. In such cases, multidetector computed tomography (MDCT) and transesophageal echocardiogram (TEE) could be performed to determine the precise dimensions of the surgical heart valves. This information should be used in conjunction with the valve True ID to determine the best transcatheter heart valve (THV). In the case of borderline sizing (i.e., 29 vs. $26 \mathrm{~mm}$ THV), it is preferable to use the larger THV where the primary pathology is $\mathrm{MR}$, and the smaller size in cases of mitral stenosis.

\section{Structural valve deterioration}

Structural dysfunction, due to progressive tissue 

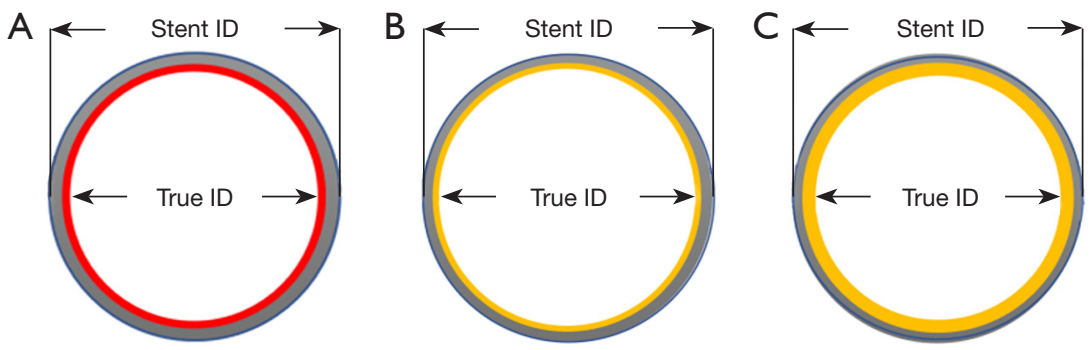

Figure 2 (A) Image depicting the true stent internal dimension and the manufacturer provided stent dimension. (B) Image showing the True ID and Stent ID for a pericardial valve with relatively larger true ID. (C) Image showing the True ID and Stent ID for a porcine prosthesis with the leaflets mounted within the stent frame. ID, internal dimension.

degeneration, is the main cause of bioprosthetic valve failure. The major pathophysiological mechanism underlying this process is leaflet calcification. This process may lead to pure stenosis due to leaflet stiffening or may also result in regurgitation due to resultant secondary micro-tears or malcoaptation (24). Recent studies have suggested that calcification of bioprosthetic valves is an active rather than a passive process that is modulated by numerous mechanisms including immune-mediated responses, lipid-mediated inflammation or dysfunctional calcium and phosphorus metabolism (25). Calcium deposits can be located on leaflet tissue or may also develop in thrombi or endocarditis vegetations (23). Glutaraldehyde valve leaflet pre-treatment is widely used to attenuate calcification and degeneration (26). Glutaraldehyde fixation cross-links and conceals the antigens, thereby making the valves immunologically inactive. However, these valves calcify over time as the glutaraldehyde alters the extracellular matrix and cellular integrity. Manufacturers of bioprosthetic valves are now using stable capping of free aldehydes and glycerolization of the animal derived valve tissue, which has been shown to substantially decrease the incidence of valvular degeneration over time (27). There is ongoing research targeted towards strategies to prevent SVD, such as raising genetically modified animals who lack xenogeneic antigens $(28,29)$.

Design-related tearing, rather than leaflet calcification, generally explains the deterioration of bovine pericardial valves (26). The formation of tissue overgrowth (e.g., pannus), thrombus or paravalvular leaks can usually explain bioprosthesis dysfunction not related to leaflet failure. Usually, valve stenosis is the consequence of calcification, pannus or thrombus formation, whereas leaflet destruction or paravalvular leak will lead to regurgitation. The outcome of degenerative tissue valves can also be a combination of stenosis and regurgitation. In mitral prosthesis, regurgitation is the predominant mechanism of valve dysfunction (49\%), followed by stenosis (21\%) and combined mechanisms (30).

The incidence of mitral bioprosthesis SVD requiring reintervention is $20 \%$ to $30 \%$ at ten years and over $50 \%$ at fifteen years. A younger age at implantation, renal failure, hyperparathyroidism, higher post-operative gradients, PPM and mitral valve position are associated with a higher risk of tissue valve deterioration (25,31-33). One of the most likely hypothesis for the greater frequency of mitral bioprosthetic failure relative to aortic bioprosthetic failure may be partially related to the higher close-off pressure in the mitral position (usually $>10$ versus $<100 \mathrm{mmHg}$ in the aortic position). Also, the closure time is expected to be greater with a mitral prosthesis compared with an aortic prosthesis, possibly contributing to a higher degeneration rate (28).

\section{Currently available transcatheter devices for failed mitral bioprostheses}

The first in-human cases of VIV and VIR procedures for mitral valve or ring dysfunction were reported in 2009 and 2011, respectively $(34,35)$. Most cases of mitral VIV and VIR have been performed with the balloon-expandable Edwards system using the Sapien S3 valve, via transapical or antegrade transfemoral (TF) approaches. The balloonexpandable Melody valve (Medtronic, Minneapolis, Minnesota) has been used in a minority of cases $(36,37)$. More recently, the use of self-expandable transcatheter valve systems for treating mitral valve dysfunction has also been reported (38).

A THV is selected based on user preference and access route. In general, only transcatheter valves with short profiles can be used in the mitral position given risk of left ventricular outflow tract (LVOT) obstruction. Currently in 
A

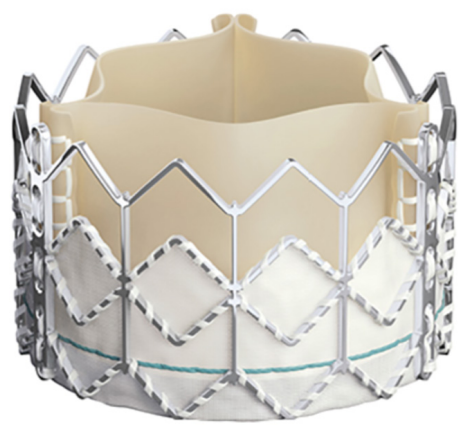

B

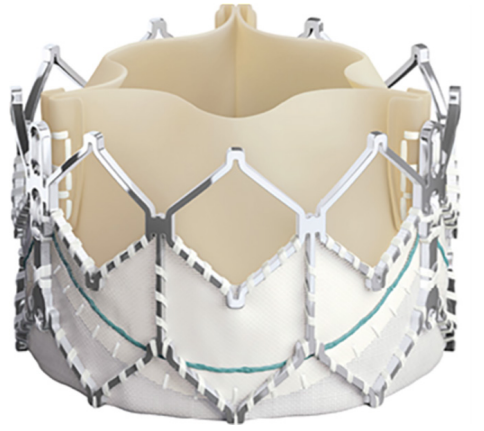

C

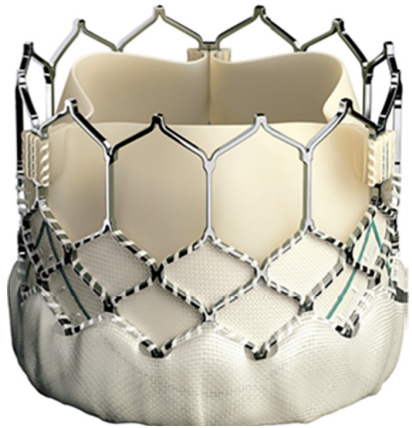

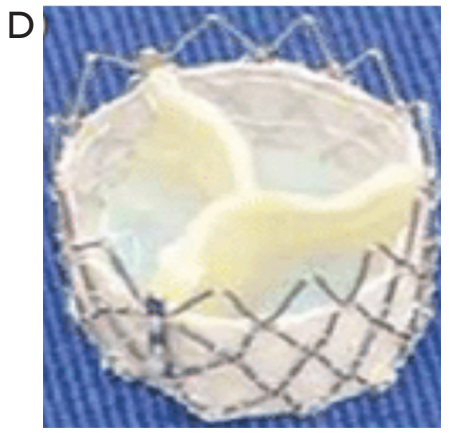
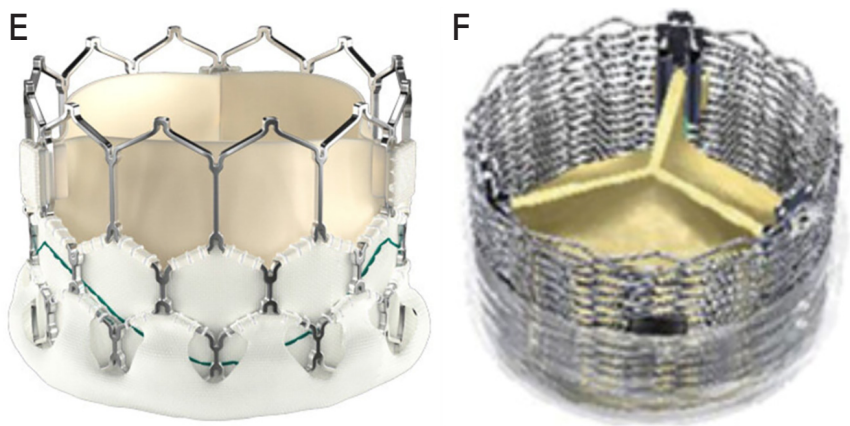

G

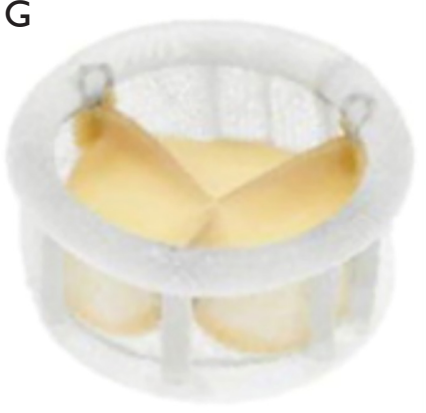

Figure 3 (A) Edwards Sapien Valve; (B) Edwards Sapien XT; (C) Edwards Sapien S3; (D) Inovare Valve; (E) Myval Valve; (F) Lotus Valve; (G) Direct Flow Valve.

the market, the most experience has been with the Edwards Lifesciences (Irvine, CA, USA) Sapien balloon expandable valves (Sapien, Sapien XT, Sapien 3). Of late, the only valve that is predominantly being used in the United States for mitral VIV and VIR is the Edwards Sapien S3 Ultra valve. Other THV devices used are Inovare, Myval, Lotus and Direct Flow (36) (Figure 3). To optimize anchoring and to limit paravalvular leakage in a mitral VIV intervention, a minimum of $2 \mathrm{~mm}$ oversizing of the transcatheter valve compared with the true internal diameter (ID) of the surgical device is preferred due to higher closing pressures and risk of embolization (37). It is not appropriate to perform extreme oversizing, as a significantly underexpanded transcatheter valve may lead to incorrect leaflet coaptation, elevated transvalvular gradient and limited durability. Under-expansion increases the risk of leaflet pin wheeling, possible thrombosis and decreases durability (39).

\section{Pre-procedural assessment}

Akin to transcatheter aortic valve replacement (TAVR) and VIV options in the aortic prosthesis realm, use of transcatheter devices in treating degenerated mitral bioprosthesis and failed mitral valve repairs with annuloplasty rings has opened a potential alternative to surgery for these patients. Experience in mitral VIV and VIR, although still limited, is on the rise. Although similar in many ways to the aortic VIV procedures, it is different with respect to patient selection, planning and procedural steps.

A thorough history detailing knowledge about native valve pathology, type of surgery and implant including the size and model and date of implantation in conjunction with information regarding additional cardiac interventions is crucial. Like aortic VIV procedures, an accurate knowledge of the surgical mitral prosthesis or ring is essential for planning a mitral VIV or VIR procedure. The first step is to identify the type and size of bioprosthesis. In the mitral position, unlike the aortic position, only stented bioprostheses are used (38). Details of the bioprosthesis can be obtained from the operative note, valve card given to the patient or from the manufacturer. In cases where information regarding the mitral valve prosthesis is not available, given that the fluoroscopic appearance of each bioprosthesis is unique, fluoroscopy guided identification of the prosthesis is very useful. Once the type of bioprosthesis is identified, the next step is to determine the true ID. As noted above, true ID is the most important dimension and determines the size of the THV to be implanted within a 
given failed bioprosthesis.

All patients should also undergo a multidisciplinary team evaluation including the interventional cardiologist, cardiologist proficient in structural imaging, cardiothoracic surgeons, cardiac anesthesiologists, nurses and possibly geriatricians and psychiatrists when appropriate.

Transthoracic echocardiography (TTE) is the first step in diagnosis of prosthetic valve dysfunction. After valve surgery, a postprocedural TTE helps in establishing the baseline prosthetic valve function. The ACC/AHA 2020 valvular heart disease guidelines recommend repeat imaging at five to ten years and then annually for follow-up of bioprosthetic heart valves, and after one year following mitral valve repair. Earlier imaging is reasonable if there are change in signs or symptoms or if early degeneration is suspected (e.g., renal failure, younger patients, diabetes, etc.). TTE provides information on change in transmitral gradients, overall cardiac function and may help in diagnosis of prosthetic valve regurgitation or stenosis. However, there are certain limitations to imaging prosthetic valves in the mitral position with TTE: (I) acoustic shadowing of the left atrium by the prosthetic stent frame may lead to an underestimation of the degree of valvular or paravalvular regurgitation, (II) poor spatial resolution may impair ability to diagnose the type of SVD.

Transesophageal echocardiography (TEE) provides excellent anatomical and hemodynamic evaluation of the bioprosthetic mitral valve. Superior spatial resolution can aid diagnosis of the etiology of SVD. The posterior position of the TEE probe allows for clear visualization of the valvular and paravalvular structures. Three-dimensional imaging is critical in evaluating prosthetic valve failure and is a crucial part of valvular assessment. Thrombi, pannus, vegetations or leaflet tears are often well visualized. Quantitative assessment of degree of MR can be done using 3D multiplanar reconstruction (MPR) and measurement of the $3 \mathrm{D}$ vena contracta area. 3D MPR also helps in measurement of the true ID. This is especially important for valves with pannus ingrowth or calcification where true ID is much smaller than what is estimated. Selection of the VIV implant size thus changes and a smaller valve may be needed. This is demonstrated in the example in Figure 4 where a $26 \mathrm{~mm}$ Sapien S3 was implanted in a $29 \mathrm{~mm}$ Carpentier-Edwards (CE) Supra-Annular Valve (SAV) due to the presence of pannus ingrowth resulting in internal dimensions of around 22-24 mm (expected true ID was $25 \mathrm{~mm}$ ).

Computed tomography (CT) is also very useful in providing information regarding valve dimensions and other geometric considerations. LVOT obstruction is one of the potential complications of mitral transcatheter valve procedures, and the proximity between the surgical valve and LVOT, as well as LVOT dimensions should also be assessed (Figures 5,6). Preprocedural CT also helps in planning of key procedural aspects such as optimal site of transseptal (Figure 7) and angle of approach for attaining coaxiality. It can be helpful to measure the angle from the long axis of the left ventricle (LV) (from the center of the mitral valve to $L V$ apex) to a perpendicular line drawn from the valve (or ring) plane. An angle of $>15^{\circ}$ for Sapien S3 ( $>20^{\circ}$ for Sapien S3 Ultra) should raise the concern for post-implant paravalvular leak (PVL) (40). Although CT scans are emerging to be an important imaging modality in these patients, characterization of different bioprosthesis based on CT scans is not standardized and thus, they can only be used as an adjunct to the sizing information in the mitral VIV application (39).

The majority of mitral VIV cases are performed within a dedicated hybrid theater or in an operating room under general anesthesia with TEE guidance and continuous hemodynamic monitoring using invasive blood pressure monitoring and pulmonary artery swan-ganz catheters (24). The team is multidisciplinary consisting of interventional cardiology, cardiothoracic surgeons, cardiac anesthesiologists, interventional echo-cardiologist with perfusionist team on standby. Given multiple team members, pre-procedure discussion with the entire team regarding the key procedural steps, monitoring and potential bailout strategies is essential to ensure best outcomes with minimal risks.

Unlike mitral VIV, mitral VIR is much more complex due to varying types of rings. Regarding the mitral rings, the D-shape of the annuloplasty ring may result in the occurrence of paravalvular leaks following transcatheter valve implantation. Because ring circularization is important to ensure efficient sealing, a transcatheter VIR procedure should be limited to deformable complete and rigid semilunar annuloplasty devices. All the known surgical mitral rings amenable to a VIR procedure include complete rings and bands (Table 1).

\section{Procedural steps}

The majority of mitral VIV or VIR procedures are performed via the TF, transseptal approach. However, there are certain circumstances in which a transapical approach may be preferred. It is also possible to implant 

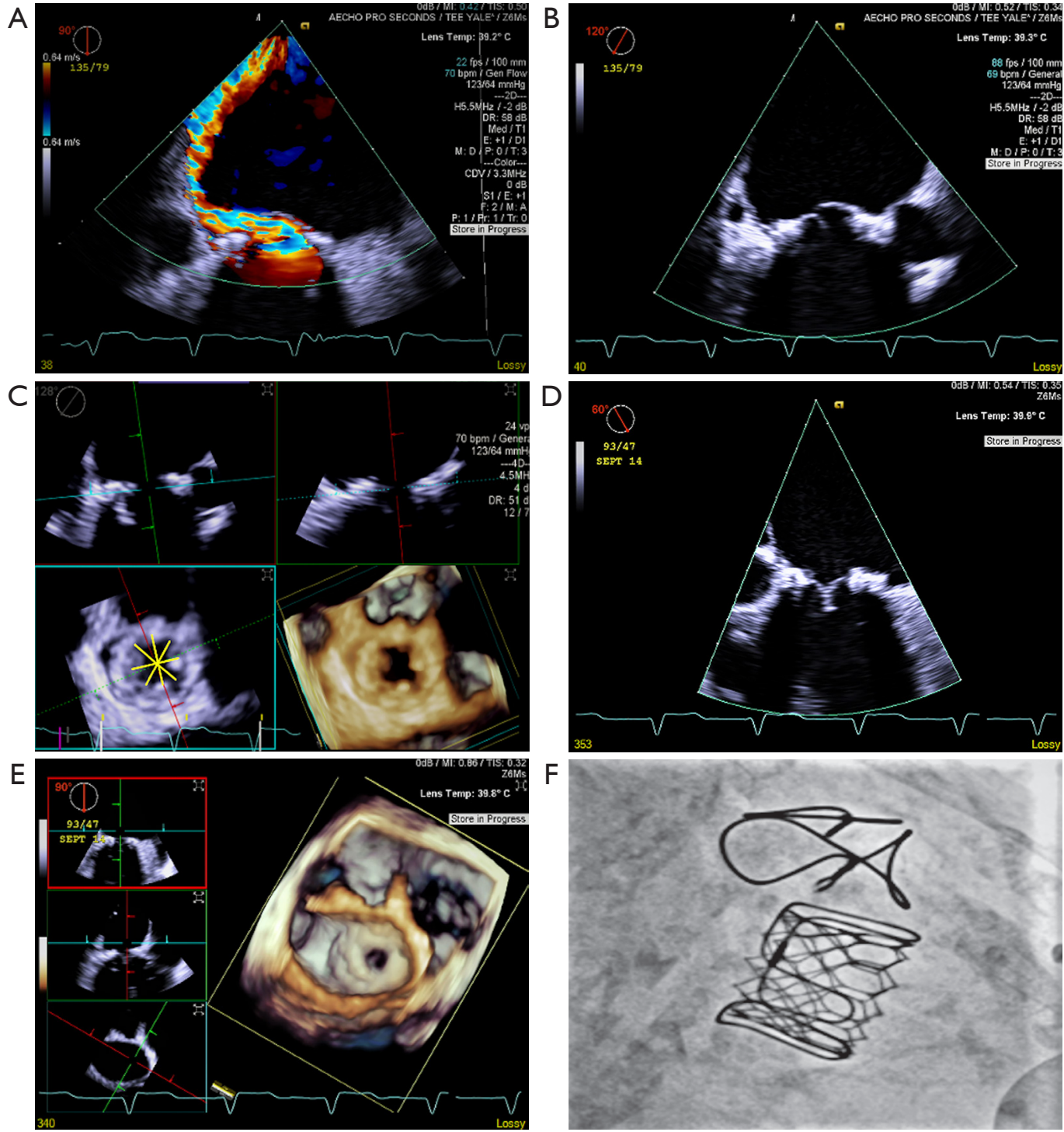

Figure 4 Example of a mitral VIV for a failed $29 \mathrm{~mm}$ CE SAV. (A) Mid-esophageal TEE view of the mitral valve in 2-chamber demonstrating severe eccentric intravalvular regurgitation. (B) Two-dimensional image demonstrating flail posteromedial leaflet (arrow). (C) 3D MPR with measurement of the internal dimension at the time of the procedure which demonstrated pannus ingrowth and dimensions ranging from 22-24 mm. (D,E) Three-dimensional en face view of the mitral valve after deployment of a $26 \mathrm{~mm}$ Sapien S3 Ultra valve. (F) Fluoroscopic image of the valve after deployment. The valve was deployed after aligning the ventricular portion of S3 and CE. VIV, valvein-valve; TEE, transesophageal echocardiogram; CE, Carpentier-Edwards; SAV, Supra-Annular Valve; MPR, multiplanar reconstruction.

the valve under direct visualization via transatrial approach either by sternotomy or right thoracotomy. Transatrial implants, though more invasive, may be necessary if there is concern for outflow tract obstruction. The benefit of an open procedure is that the leaflets of the prosthetic valve can be excised, thus allowing blood to flow through the THV frame in systole. Without excision, the old leaflets are stretched around the outside of the THV frame, in essence creating a tube graft that can obstruct LVOT flow. This is also a risk in VIR. A percutaneous approach to lacerate a potentially obstructive leaflet has been pioneered (LAMPOON), but experience is limited (41).

TF transseptal approach is the preferred technique for VIV and VIR implants. The only commercially approved 

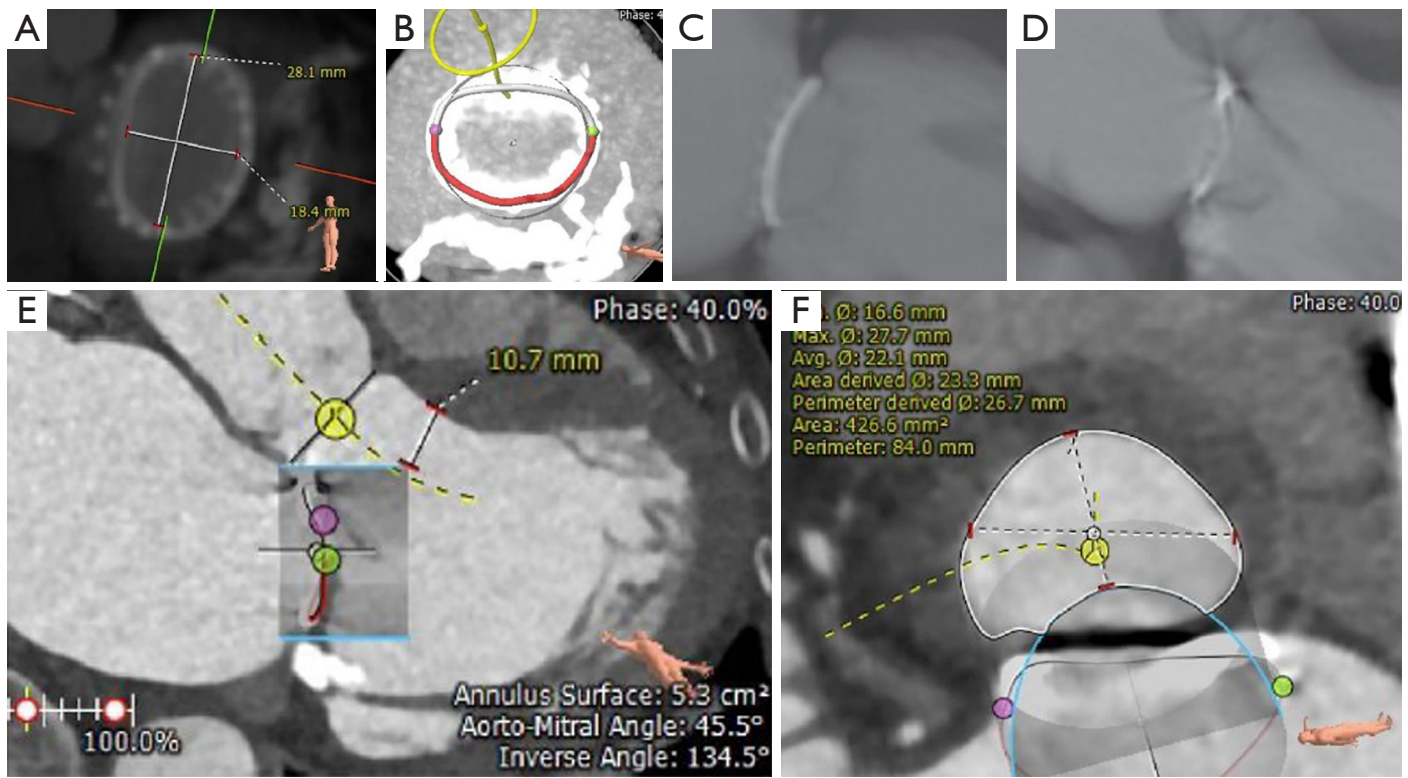

Figure 5 Evaluation for VIR using 3Mensio (Pie Medical Imaging, The Netherlands) CT scan analysis for a failed 30 mm Edwards Physio II complete ring (A,B). Note the pronounced saddle shape of the ring (C,D). Image (E) demonstrates the ideal position of the proposed THV, a $29 \mathrm{~mm}$ Sapien S3. The inflow of the THV would be $10.7 \mathrm{~mm}$ away from the LVOT, suggesting the neo-LVOT should be sufficiently large. Image (F) shows the neo-LVOT in cross section at its smallest area, measuring $426 \mathrm{~mm}^{2}$, well above the cutoff of $120 \mathrm{~mm}^{2}$. Thus, the risk of outflow tract obstruction is low. VIR, valve-in-ring; THV, transcatheter heart valve; LVOT, left ventricular outflow tract.
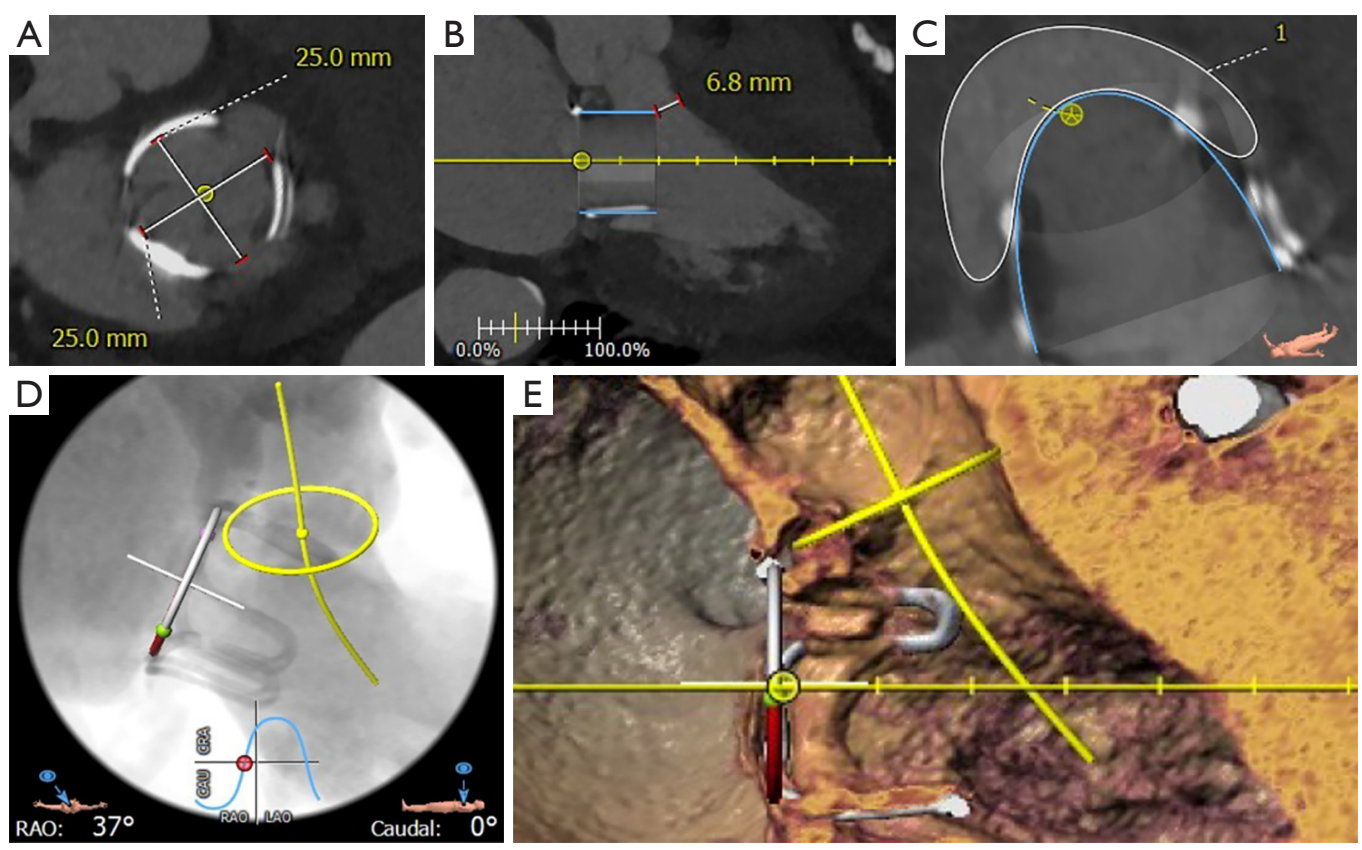

Figure 6 Preprocedural assessment cardiac CT in a patient with a failed $29 \mathrm{~mm}$ CE SAV. The internal diameter of the valve is $25 \mathrm{~mm}$ at the level of the annulus (A). A $26 \mathrm{~mm}$ Sapien S3 virtual valve was placed using 3Mensio (Pie Medical Imaging) which demonstrates $6.8 \mathrm{~mm}$ of clearance from the interventricular septum (B) and a neo-LVOT area of $312 \mathrm{~mm}^{2}(\mathrm{C})$. A deployment angle $\left(\mathrm{RAO} 37^{\circ}\right.$, Caudal $\left.0^{\circ}\right)$ is determined by aligning the inflow of the SAV (D), as well as the aorto-mitral angle $\left(114^{\circ}\right)$ (E). CE, Carpentier-Edwards; SAV, Supra-Annular Valve; LVOT, left ventricular outflow tract. 

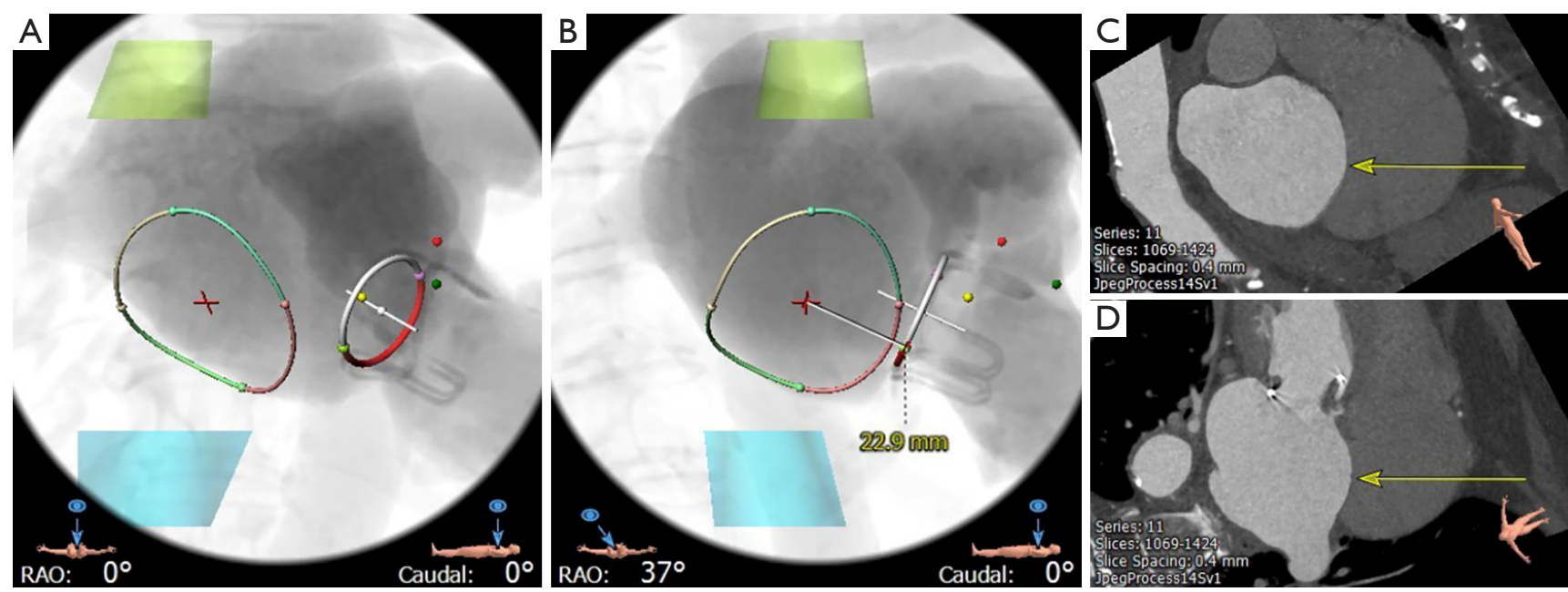

Figure 7 Transseptal planning using 3Mensio analysis of CT scan. A virtual puncture site can be identified that is central on the fossa. In the AP projection (A), the site should line up with the lowest portion of the SAV inflow, and ideally no more superior than the mid portion of the SAV. The coplanar angle can be used to determine the height of the puncture site above the valve annulus (B). A height of 2.5-3.5 cm is desirable, and more height can be gained by puncturing more posteriorly. Two-dimensional imaging can also be used to visualize the puncture site (C,D). There was a large degree of valve pannus formation in this valve (D). SAV, Supra-Annular Valve.

TF system in the United States is the Edwards Sapien device, and the following discussion will focus on this approach. After perclose of the right femoral vein (Perclose ProGlide, Abbott), serial dilators are used to upsize to the Edwards e-sheath (14 or $16 \mathrm{Fr}$ ) or a $24 \mathrm{Fr}$ sheath. Our preference is the GORE DrySeal ( $24 \mathrm{Fr}, 33 \mathrm{~cm}$ long) given its excellent hemostatic valve. Using this larger sheath allows for the Edwards Sapien valve to be mounted directly on the balloon, which reduces the amount of catheter that needs to extend beyond the sheath tip during final preparation of the valve and delivery system. If the valve is mounted in the traditional location (used for aortic procedures), the nosecone of the delivery system may protrude well across the intra-atrial septum to advance the balloon from the sheath. Special note needs to be made of the valve that is mounted "upside down" when compared to a TF TAVR case.

After placement of the large bore sheath, a transseptal puncture is performed. Our preference is to use an SL1 sheath with a Baylis needle. The Baylis needle uses radiofrequency energy to puncture the septum. It is important to note whether the interatrial septum was intervened upon during the initial mitral valve surgery. It may be challenging to introduce devices across the septal puncture site if the area has been patched or over sewn. Performing balloon dilation of the site (described below) will facilitate the delivery of equipment (Figure 8). The preferred location for the transseptal puncture is guided both by fluoroscopy and TEE. With the image intensive intensifier in the AP view, the height of the transseptal puncture site should be at the most caudal portion of the sewing ring. Puncturing slightly higher than this may be beneficial in patients who have a relatively flat aorto-mitral angle, allowing for a more coaxial alignment of the new valve.

Following transseptal a puncture, the pigtail Baylis needle is placed in the left atrium (LA). At this point our preference is to exchange the transseptal sheath and dilator for an $8.5 \mathrm{Fr}$ (medium curve) steerable Agilis $\mathrm{NxT}$ sheath (Abbott). This allows for more control and support when attempting to cross the surgical valve as well as delivering the stiff wire. We then use a balloon wedge catheter (balloon inflated in the LA with $\mathrm{CO}_{2}$ ) or a pigtail catheter to cross the mitral valve. If a balloon wedge catheter is used, it needs to be exchanged for a pigtail using a long 035J wire. Over the pigtail, a stiff guidewire can be introduced. Our preference is a Confida guidewire $(260 \mathrm{~cm}$, Medtronic) in most cases. A stiffer wire (e.g., Lunderquist Wire, Cook Medical) may put too much pressure on the lateral wall of the LV. The delivery system may prolapse towards the outer wall of the LA if a more flexible wire is used (e.g., Amplatz Wire, Boston Scientific). When placing the stiff guide wire in the $\mathrm{LV}$, it is important to check that the wire is free from 

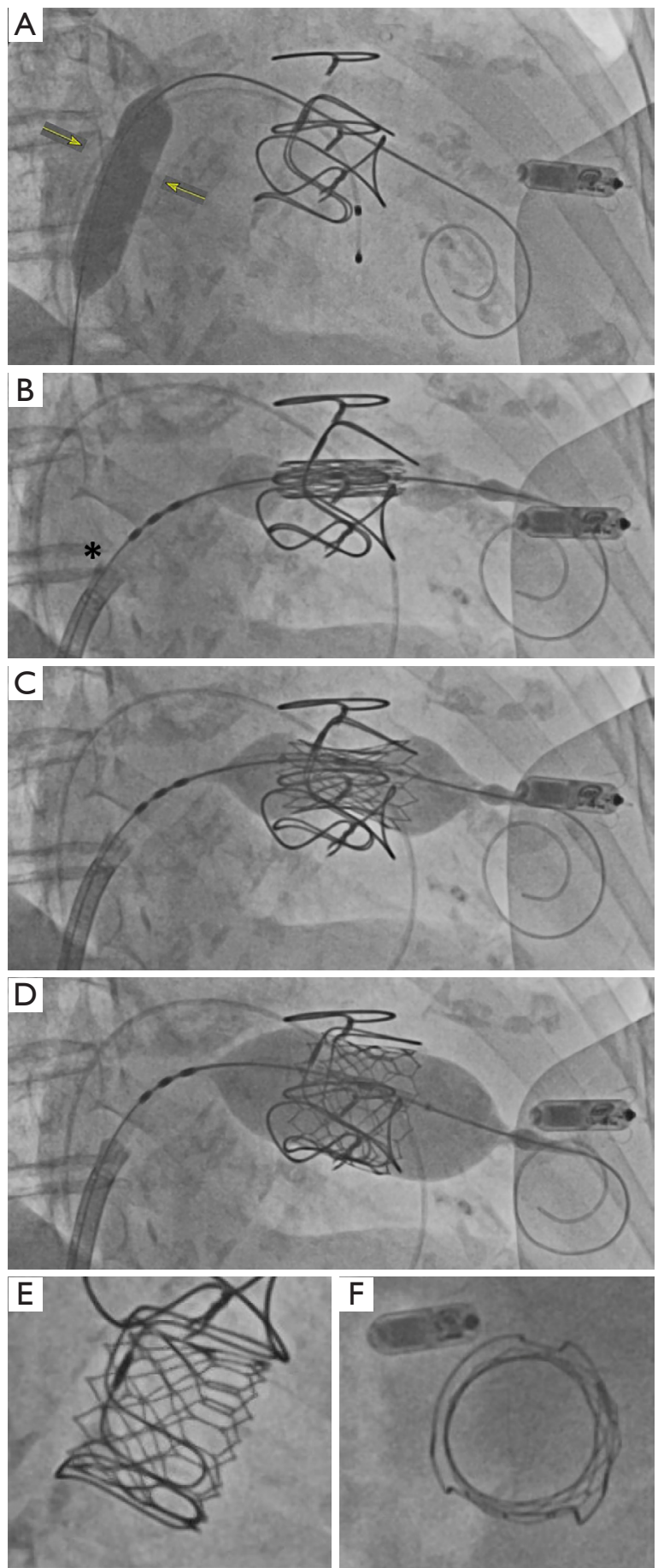

Figure 8 Deployment sequence of a $26 \mathrm{~mm}$ Sapien S3 in a $29 \mathrm{~mm}$ CE SAV. The mechanism of failure of the SAV was prolapse of two cusps due to leaflet degeneration resulting in severe MR. After delivery of the Confida guidewire, the septal puncture site (yellow arrows) was dilated with a $14 \times 40 \mathrm{~mm}$ balloon (A). The valve is delivered into position (B) and the pusher is withdrawn more proximally than typical ${ }^{[*}$ to increase flexibility of the system during deployment. As the THV balloon is inflated, the valve self-centers as it anchors on the SAV facilitated by the operator in position one applying forward pressure on the system (C,D). Coplanar and en face images of the VIV are shown in images (E,F). (Note: patient also has a Micra leadless pacemaker in the apex of the right ventricle). CE, Carpentier-Edwards; SAV, Supra-Annular Valve; VIV, valve-in-valve; THV, transcatheter heart valve. 
the subvalvular apparatus using TEE.

Over the stiff guidewire, a $14 \times 40 \mathrm{~mm}$ balloon $(100 \mathrm{~cm}$ shaft, 0.035 inch) is introduced. In cases of prosthetic mitral stenosis, we advanced the balloon to first predilate the valve. The same balloon is then retracted and used to dilate the intra-atrial septum. The balloon is exchanged and the Edwards Commander Delivery System is introduced with the Edwards logo facing down (180 degrees from usual). If the valve is mounted as described on the balloon, the balloon catheter does not need to be pulled back as the first step. Rather, the fine adjustment wheel can be used to direct the valve into position between the two Valve Alignment Markers on the Flex Catheter. The system is then advanced with progressive addition of flex on the catheter (usually reaching roughly two-thirds of full flex when crossing the septum). When the valve has crossed the mitral annulus, the valve "pusher" can be retracted. It is often helpful to retract the pusher further back than usual as that causes the shaft to be more flexible and facilitates a coaxial implant during balloon inflation.

The appropriate valve position depends on the type of surgical valve that is in place. Broadly speaking, the valve should be deployed with $10 \%$ in the left atrium and $90 \%$ in the LV (Figure 8). For VIR, note that that many rings are "saddle shaped" and thus will not have a flat annular plane. The most atrial portions of the saddle should be used as a visual reference for the implant (with the goal of $10 \%$ of the THV being on the atrial side of this reference point). There are references available to identify fluoroscopic landmarks for different types of surgical valves and rings to ensure proper deployment depth (VIV Mitral application, version 2.2, UBQO Limited).

Given the angle of approach from the septal puncture site to the valve annulus, the new valve will not be coaxial to the surgical valve or ring on initial positioning. Maneuvers that can improve alignment usually involve manipulation of both the wire and the balloon catheter. Advancing the wire into the LV while advancing the delivery system forward will tilt inflow of the valve laterally as the system moves forward. Caution should be used to prevent excessive pressure of the guidewire on the lateral wall of the LV, particularly if the patient has increased risk factors for myocardial perforation (e.g., cardiomyopathy, chronic steroid use, advanced age). Some implanters have inserted a 6 Fr sheath percutaneously near the LV apex to externalize the guidewire and improve coaxial implantation.

The deployment of the valve does not necessarily require rapid ventricular pacing. However, in our experience, this provides some additional confidence that there will be minimal motion during deployment. The inflation of the deployment balloon should be exceptionally slow to allow for the valve to be self-centered and become more coaxial on final deployment. As the balloon is inflated and the THV begins to anchor on the surgical valve, forward pressure on the delivery system will promote the inflow of the valve to translate laterally without advancing further into the LV. We deploy these valves at relatively high pressures (8-10 atm) given the risk of annular rupture is low and a higher pressure deployment will help to flare the LV side of the valve and minimize the risk of valve embolization into the LA during LV systole. Once the delivery balloon is fully deflated, the system should be carefully withdrawn into the left atrium with special care to prevent the nosecone from interacting with the outflow of the THV.

Immediate post-deployment evaluation of the THV using TEE should be performed, evaluating leaflet function, valvular regurgitation (paravalvular or central) and LVOT velocities. The guide wire will likely be impinging on the function of one of the leaflets given how it tracks tangentially cross the valve. When it has been determined that post-dilation or deployment of the second valve is not necessary, the delivery system can be removed from the body and the guidewire can be exchanged for a pigtail. The pigtail can be removed using a long J-wire after a full TEE assessment is made.

The use of a transapical approach is more invasive but can be considered if transseptal approach is not possible. The distinct advantage of this approach is that the delivery catheter is coaxial to the mitral annular plane once inserted. Furthermore, there is greater control of valve position during deployment given the short delivery system and straight trajectory of the system.

\section{Complications}

Access complications for the femoral approach are less frequent when compared with TAVR given venous access. Failed Percloses can be managed by placement of an additional Perclose, though given the large sheath size this may not be possible. A sheath can be replaced and pulled manually when the activated clotting time normalizes post procedure. A figure of eight stitch can also be placed over the site when needed. A Femostop (Abbott) can be utilized at low pressure $(20-30 \mathrm{mmHg})$ if there is persistent 
bleeding from the site.

Transseptal complications are rare, but depending on the site of injury, may require open surgical repair. We typically only give half of the full heparin dose prior to transseptal puncture, and only administer the remaining heparin after the transseptal is performed and TEE demonstrates no pericardial effusion. We also transduce the pressure waveform from the transseptal needle to help verify crossing from the right atrium (RA) to LA. If at any point there is concern that the transseptal system is not in the LA or RA, the exact position should be verified before moving the device as the system may be providing hemostasis at the errant puncture site.

Mechanical injury to the $\mathrm{LV}$ myocardium is a known risk of this procedure due to interaction with the guidewire. An excessive amount of force can inadvertently be placed on the guidewire in an attempt to align the valve. Throughout the cardiac cycle there is repetitive linear injury to the endomyocardial border of the left ventricle that can result in a linear laceration. Ultimately, a hematoma forms and hydraulic forces can cause the hematoma to expand within the muscle, creating a non-uniform intramyocardial injury that ultimately extends into the pericardial space. This often results in a rapidly accumulating pericardial effusion (seen on TEE) and hemodynamic collapse. Angiogram of the LV will show contrast extravasation into the pericardial space. Pericardiocentesis is required in addition to reversal of heparin (blood can be auto-transfused until protamine is given). Blood transfusion should be initiated and preparations for conversion to open surgical repair should be made. Surgical repair of this myocardial injury is very challenging and rarely successful given nature of the injury.

As mentioned above, LVOT obstruction can occur when the surgical valve leaflets (or native leaflet, in the case of VIR) drape over the outer frame of the THV. If high LVOT gradients are noted, treatment includes volume loading, beta blockade and increasing afterload (without increasing chronotropy or inotropy). An alcohol septal ablation can be considered for LVOT obstruction in selected patients who do not respond to the more conservative measures.

Valve embolization can occur during deployment. VIR procedures have a higher risk of embolization into the $\mathrm{LV}$ given a narrower landing zone. If the valve embolizes into the LA or LV, the wire should be left in place to keep the valve relatively stationary while preparations can be made for conversion to open surgery. If there is significant PVL due to a valve that is implanted at the improper depth (too shallow or deep) or too canted, the best course of action is to promptly insert a second valve of the same size but at the proper depth.

\section{Conclusions}

Percutaneous transcatheter options have been increasingly employed in patients with failed mitral prostheses deemed high risk for redo surgery. Although there have been few case studies demonstrating the safety and efficacy of TEER in patients with mitral valve repair failure (42), the success of TMVR in high risk patients with prior surgeries has been well documented. The multicenter TMVR and Transcatheter Valve Therapy (TVT) registries have recorded over a combined 2,000 cases of patients undergoing VIV or VIR with TMVR (43-45). These cases represent a high risk patient cohort with a mean Society of Thoracic Surgeons (STS) score of approximately 9-10\%. Majority of these cases were performed via a transapical approach with the Sapien platform being a heavy favorite accounting for over $90 \%$ of the cases. The TMVR and TVT registries demonstrate an excellent rate of success for mitral VIV, with technical success rates over $90 \%$ and procedural success rates close to $75 \%$. All-cause mortality at 30 days was $5.4 \%$ to $8.1 \%$, increasing to $14.0 \%$ to $16.7 \%$ at one year. Notably, transapical access was associated with higher all-cause mortality at one year compared with transseptal access (21.7\% versus $15.8 \%)$ in the TVT registry (45). The high rates of technical and procedural success in the TVT registry were reflected in significant clinical improvements at one year, with only $9.7 \%$ of the patients reporting class III/IV heart failure symptoms, down from $87.1 \%$ pre-procedure.

Mitral VIR procedures do not achieve the same rates of success as VIV, with reported technical success rates of $80.9 \%$ to $82.9 \%$ and procedural success rates of only $57.4 \%$. Patients undergoing a VIR procedure also have higher rates of all-cause mortality both at 30 days $(\sim 10 \%)$ and at one year ( 30\%) (43). Overall, patients undergoing TMVR with failed annuloplasty rings and severe mitral annular calcification are nearly twice as likely to die compared to patients undergoing VIV procedures. There is no statistically significant difference in rates of stroke, bleeding, acute kidney injury and major vascular complications. Despite the lower success and higher complication rates for VIR, TMVR may be the more attractive option in these patients rather than redo surgery with TMVR patients 
showing equivalent rates of mortality compared to surgical MVR patients, despite having higher STS scores (46).

\section{Future directions}

Mitral VIV and VIR therapies, up until this point, have been adapted from TAVR systems. There is no commercially approved device in the United States that is exclusively designed for the mitral position. Future versions of a VIV/VIR device will most likely be based on TF TMVR platforms being developed for native mitral valve replacement. There are ongoing trials for transapical TMVR devices (e.g., APOLLO, Tendyne), some of which are being adapted to a TF system. Though native TMVR valves are quite large (accommodating native annuli from 42 to $48 \mathrm{~mm}$ in diameter), a small implant for VIV or VIR could be envisioned that can be delivered using the same delivery system. TF TMVR systems under development prioritize the ability to articulate the delivery catheter in such a way that optimizes coaxial alignment and depth control, two features that will address two primary shortcomings of the current platform.

\section{Acknowledgments}

Funding: None.

\section{Footnote}

Conflicts of Interest: The authors have no conflicts of interest to declare.

Open Access Statement: This is an Open Access article distributed in accordance with the Creative Commons Attribution-NonCommercial-NoDerivs 4.0 International License (CC BY-NC-ND 4.0), which permits the noncommercial replication and distribution of the article with the strict proviso that no changes or edits are made and the original work is properly cited (including links to both the formal publication through the relevant DOI and the license). See: https://creativecommons.org/licenses/by-nc-nd/4.0/.

\section{References}

1. Virani SS, Alonso A, Benjamin EJ, et al. Heart Disease and Stroke Statistics-2020 Update: A Report From the American Heart Association. Circulation 2020;141:e139-596.

2. Iung B, Baron G, Butchart EG, et al. A prospective survey of patients with valvular heart disease in Europe: The Euro Heart Survey on Valvular Heart Disease. Eur Heart J 2003;24:1231-43.

3. Nkomo VT, Gardin JM, Skelton TN, et al. Burden of valvular heart diseases: a population-based study. Lancet 2006;368:1005-11.

4. Dziadzko V, Clavel MA, Dziadzko M, et al. Outcome and undertreatment of mitral regurgitation: a community cohort study. Lancet 2018;391:960-9.

5. Chatterjee S, Rankin JS, Gammie JS, et al. Isolated mitral valve surgery risk in 77,836 patients from the Society of Thoracic Surgeons database. Ann Thorac Surg 2013;96:1587-94; discussion 1594-5.

6. Goldstone AB, Chiu P, Baiocchi M, et al. Mechanical or Biologic Prostheses for Aortic-Valve and Mitral-Valve Replacement. N Engl J Med 2017;377:1847-57.

7. Chiang YP, Chikwe J, Moskowitz AJ, et al. Survival and long-term outcomes following bioprosthetic vs mechanical aortic valve replacement in patients aged 50 to 69 years. JAMA 2014;312:1323-9.

8. Akins CW, Buckley MJ, Daggett WM, et al. Risk of reoperative valve replacement for failed mitral and aortic bioprostheses. Ann Thorac Surg 1998;65:1545-51; discussion 1551-2.

9. Lytle BW, Cosgrove DM, Taylor PC, et al. Reoperations for valve surgery: perioperative mortality and determinants of risk for 1,000 patients, 1958-1984. Ann Thorac Surg 1986;42:632-43.

10. Bortolotti U, Milano A, Mossuto E, et al. Early and late outcome after reoperation for prosthetic valve dysfunction: analysis of 549 patients during a 26-year period. J Heart Valve Dis 1994;3:81-7.

11. Lazam S, Vanoverschelde JL, Tribouilloy C, et al. TwentyYear Outcome After Mitral Repair Versus Replacement for Severe Degenerative Mitral Regurgitation: Analysis of a Large, Prospective, Multicenter, International Registry. Circulation 2017;135:410-22.

12. Goldstein D, Moskowitz AJ, Gelijns AC, et al. Two-Year Outcomes of Surgical Treatment of Severe Ischemic Mitral Regurgitation. N Engl J Med 2016;374:344-53.

13. Feldman T, Foster E, Glower DD, et al. Percutaneous repair or surgery for mitral regurgitation. $\mathrm{N}$ Engl J Med 2011;364:1395-406.

14. Feldman T, Kar S, Elmariah S, et al. Randomized Comparison of Percutaneous Repair and Surgery for Mitral Regurgitation: 5-Year Results of EVEREST II. J Am Coll Cardiol 2015;66:2844-54.

15. Sorajja P, Vemulapalli S, Feldman T, et al. Outcomes With 
Transcatheter Mitral Valve Repair in the United States: An STS/ACC TVT Registry Report. J Am Coll Cardiol 2017;70:2315-27.

16. Stone GW, Lindenfeld J, Abraham WT, et al. Transcatheter Mitral-Valve Repair in Patients with Heart Failure. N Engl J Med 2018;379:2307-18.

17. Otto CM, Nishimura RA, Bonow RO, et al. 2020 ACC/ AHA Guideline for the Management of Patients With Valvular Heart Disease: A Report of the American College of Cardiology/American Heart Association Joint Committee on Clinical Practice Guidelines. Circulation 2021;143:e72-e227.

18. Bothe W, Miller DC, Doenst T. Sizing for mitral annuloplasty: where does science stop and voodoo begin? Ann Thorac Surg 2013;95:1475-83.

19. Christakis GT, Buth KJ, Goldman BS, et al. Inaccurate and misleading valve sizing: a proposed standard for valve size nomenclature. Ann Thorac Surg 1998;66:1198-203.

20. Gurvitch R, Cheung A, Ye J, et al. Transcatheter valve-invalve implantation for failed surgical bioprosthetic valves. J Am Coll Cardiol 2011;58:2196-209.

21. Bapat V, Mydin I, Chadalavada S, et al. A guide to fluoroscopic identification and design of bioprosthetic valves: a reference for valve-in-valve procedure. Catheter Cardiovasc Interv 2013;81:853-61.

22. Bapat V. Valve-in-valve apps: why and how they were developed and how to use them. EuroIntervention 2014;10 Suppl U:U44-51.

23. Bapat VN, Attia R, Thomas M. Effect of valve design on the stent internal diameter of a bioprosthetic valve: a concept of true internal diameter and its implications for the valve-in-valve procedure. JACC Cardiovasc Interv 2014;7:115-27.

24. Paradis JM, Del Trigo M, Puri R, et al. Transcatheter Valve-in-Valve and Valve-in-Ring for Treating Aortic and Mitral Surgical Prosthetic Dysfunction. J Am Coll Cardiol 2015;66:2019-37.

25. Mahjoub H, Mathieu P, Larose E, et al. Determinants of aortic bioprosthetic valve calcification assessed by multidetector CT. Heart 2015;101:472-7.

26. Schoen FJ, Levy RJ. Calcification of tissue heart valve substitutes: progress toward understanding and prevention. Ann Thorac Surg 2005;79:1072-80.

27. Shang H, Claessens SM, Tian B, et al. Aldehyde reduction in a novel pericardial tissue reduces calcification using rabbit intramuscular model. J Mater Sci Mater Med 2017;28:16.

28. Kostyunin AE, Yuzhalin AE, Rezvova MA, et al.
Degeneration of Bioprosthetic Heart Valves: Update 2020. J Am Heart Assoc 2020;9:e018506.

29. Smood B, Hara H, Cleveland DC, et al. In Search of the Ideal Valve: Optimizing Genetic Modifications to Prevent Bioprosthetic Degeneration. Ann Thorac Surg 2019;108:624-35.

30. Ribeiro AH, Wender OC, de Almeida AS, et al. Comparison of clinical outcomes in patients undergoing mitral valve replacement with mechanical or biological substitutes: a 20 years cohort. BMC Cardiovasc Disord 2014;14:146.

31. Poirer NC, Pelletier LC, Pellerin M, et al. 15-year experience with the Carpentier-Edwards pericardial bioprosthesis. Ann Thorac Surg 1998;66:S57-61.

32. Ruel M, Kulik A, Rubens FD, et al. Late incidence and determinants of reoperation in patients with prosthetic heart valves. Eur J Cardiothorac Surg 2004;25:364-70.

33. Johnston DR, Soltesz EG, Vakil N, et al. Long-term durability of bioprosthetic aortic valves: implications from 12,569 implants. Ann Thorac Surg 2015;99:1239-47.

34. Cheung A, Webb JG, Wong DR, et al. Transapical transcatheter mitral valve-in-valve implantation in a human. Ann Thorac Surg 2009;87:e18-20.

35. de Weger A, Ewe SH, Delgado V, et al. First-inman implantation of a trans-catheter aortic valve in a mitral annuloplasty ring: novel treatment modality for failed mitral valve repair. Eur J Cardiothorac Surg 2011;39:1054-6.

36. Cullen MW, Cabalka AK, Alli OO, et al. Transvenous, antegrade Melody valve-in-valve implantation for bioprosthetic mitral and tricuspid valve dysfunction: a case series in children and adults. JACC Cardiovasc Interv 2013;6:598-605.

37. Kliger C, Angulo R, Maranan L, et al. Percutaneous complete repair of failed mitral valve prosthesis: simultaneous closure of mitral paravalvular leaks and transcatheter mitral valve implantation - single-centre experience. EuroIntervention 2015;10:1336-45.

38. Dvir D. Transcatheter mitral valve-in-valve and valve-inring implantations. Euro-PCR. 2015.

39. Pirelli L, Hong E, Steffen R, et al. Mitral valve-invalve and valve-in-ring: tips, tricks, and outcomes. Ann Cardiothorac Surg 2021;10:96-112.

40. Greenbaum AB, Lisko JC, Gleason PT, et al. Annularto-Apical "Emory Angle" to Ensure Coaxial Mitral Implantation of the SAPIEN 3 Valve. JACC Cardiovasc Interv 2020;13:2447-50.

41. Khan JM, Babaliaros VC, Greenbaum AB, et al. Anterior 
Leaflet Laceration to Prevent Ventricular Outflow Tract Obstruction During Transcatheter Mitral Valve Replacement. J Am Coll Cardiol 2019;73:2521-34.

42. Grasso C, Ohno Y, Attizzani GF, et al. Percutaneous mitral valve repair with the MitraClip system for severe mitral regurgitation in patients with surgical mitral valve repair failure. J Am Coll Cardiol 2014;63:836-8.

43. Yoon SH, Whisenant BK, Bleiziffer S, et al. Outcomes of transcatheter mitral valve replacement for degenerated bioprostheses, failed annuloplasty rings, and mitral annular calcification. Eur Heart J 2019;40:441-51.

44. Guerrero M, Vemulapalli S, Xiang Q, et al. Thirty-Day Outcomes of Transcatheter Mitral Valve Replacement for Degenerated Mitral Bioprostheses (Valve-in-Valve),
Failed Surgical Rings (Valve-in-Ring), and Native Valve With Severe Mitral Annular Calcification (Valve-in-Mitral Annular Calcification) in the United States: Data From the Society of Thoracic Surgeons/American College of Cardiology/Transcatheter Valve Therapy Registry. Circ Cardiovasc Interv 2020;13:e008425.

45. Whisenant B, Kapadia SR, Eleid MF, et al. One-Year Outcomes of Mitral Valve-in-Valve Using the SAPIEN 3 Transcatheter Heart Valve. JAMA Cardiol 2020;5:1245-52.

46. Kamioka N, Babaliaros V, Morse MA, et al. Comparison of Clinical and Echocardiographic Outcomes After Surgical Redo Mitral Valve Replacement and Transcatheter Mitral Valve-in-Valve Therapy. JACC Cardiovasc Interv 2018;11:1131-8.
Cite this article as: Agarwal V, Kaple RK, Mehta HH, Singh P, Bapat VN. Current state of transcatheter mitral valve implantation in bioprosthetic mitral valve and in mitral ring as a treatment approach for failed mitral prosthesis. Ann Cardiothorac Surg 2021;10(5):585-604. doi: 10.21037/acs-2021tviv-80 